\title{
Lung Myoepithelial Carcinoma
}

National Cancer Institute

\section{Source}

National Cancer Institute. Lung Myoepithelial Carcinoma. NCI Thesaurus. Code C142830.

A very rare malignant lung tumor with myoepithelial differentiation. 